\title{
What is Localization?
}

\author{
R. del Rio \\ IIMAS-UNAM, Apdo. Postal 20-726, Admon. No. 20, OI000 Mexico, D.F., Mexico \\ S. Jitomirskaya \\ Department of Mathematics, University of California at Irvine, Irvine, California 92717 \\ Y. Last and B. Simon \\ Division of Physics, Mathematics, and Astronomy, California Institute of Technology 253-37, Pasadena, California 91125 \\ (Received 22 March 1995)
}

\begin{abstract}
We examine various issues relevant to localization in the Anderson model. We show there is more to localization than exponentially localized states by presenting an example with such states but where $\left\langle x(t)^{2}\right\rangle / t^{2-\delta}$ is unbounded for any $\delta>0$. We show that the recently discovered instability of localization under rank one perturbations is only a weak instability.
\end{abstract}

PACS numbers: $72.15 . \mathrm{Rn}$

Localization in random media is basic to a variety of physical situations. We wish to report here on a number of rigorous mathematical results that shed light on the phenomenon of localization in the Anderson model. Mathematically complete proofs of our results will appear elsewhere [1]. Our goal here is to describe the ideas behind the results.

Throughout, we will consider the Anderson model, that is, the Hamiltonian $H_{\omega}$ on $\ell^{2}\left(Z^{d}\right)$ (namely, on the $d$ dimensional cubic lattice)

$$
\left(H_{\omega} u\right)(n)=\sum_{|j|=1} u(n+j)+V_{\omega}(n) u(n)
$$

where the potentials $V_{\omega}$ are identically distributed independent random variables with distribution [2]

$$
\frac{1}{2 \eta} \chi_{[-\eta, \eta]}(x) d x,
$$

with $\chi[-\eta, \eta]$ the characteristic function of the interval $[-\eta, \eta]$.

Many of the claimed proofs of localization show that, for almost all $\omega$, an Anderson model Hamiltonian $H_{\omega}$ has a complete set of normalized eigenvectors [3] $\left\{\varphi_{\omega, m}\right\}_{m=1}^{\infty}$ obeying

$$
\left|\varphi_{\omega, m}(n)\right| \leq C_{\omega, m} e^{-A\left|n-n_{\omega, m}\right|},
$$

where $A$ is fixed, the $n_{\omega, m}$ 's are some centers of localization, and the $C_{\omega, m}$ 's are constants depending on $\omega$ and $m$.

Our first result is an example that shows that mere "exponential localization" of eigenfunctions in the form of Eq. (2) need not have very strong consequences for the dynamics. We can construct a nonrandom potential $V$ in one dimension with the following: (i) $H$ has a complete set of normalized eigenvectors obeying Eq. (2). (ii) Let $\left\langle x^{2}\right\rangle(t)$ denote $\left\langle e^{-i t H} \delta_{0}, x^{2} e^{-i t H} \delta_{0}\right\rangle$; then for any $\delta>0$, $\left\langle x^{2}(t)\right\rangle / t^{2-\delta}$ is unbounded as $t \rightarrow \pm \infty[4]$.

The potential $V$ for this example is

$$
V(n)=3 \cos (2 \pi \alpha n+\theta)+\lambda \delta_{n 0},
$$

which we consider on the positive half of the lattice ( $n \geq$ 0 ), with a Dirichlet (or any other) boundary condition at the origin. The 3 in front of the cosine can be replaced by any number larger than 2 , and is chosen so that when $\lambda=0$ the problem has a positive Lyapunov exponent [5]. The $\alpha$ is an irrational, which is specially chosen so that for suitable time scales $T_{n} \rightarrow \infty, V$ is so close to periodic that we can show $\left\langle x^{2}\left(T_{n}\right)\right\rangle$ is large compared to $T_{n}^{2-\delta}$. The local perturbation $\lambda \delta_{n 0}$ pushes the spectrum to be pure point and forces Eq. (2) to hold.

While $V$ is very far from random, it illustrates that Eq. (2) is not enough to restrict dynamics. The main failing in (2) is the total freedom given to the constants $C_{\omega, m}$. Indeed, when one thinks of "localization," one usually thinks of the eigenvectors as being confined, at least roughly, within some typical length scale. If the $C_{\omega, m}$ 's are allowed to grow arbitrarily as $m$ changes, it means that eigenvectors are allowed to be "extended" over arbitrarily large length scales. We have shown that a correct condition, which does give correspondence between eigenvector localization and dynamical localization, is what we call semiuniformly localized eigenvectors (SULE): There are sites $n_{\omega, m}$ so that for each $\epsilon>0$ there is $C_{\omega, \epsilon}$ for which

$$
\left|\varphi_{\omega, m}(n)\right| \leq C_{\omega, \epsilon} e^{\epsilon\left|n_{\omega, m}\right|} e^{-A\left|n-n_{\omega, m}\right|} .
$$

Condition (3) says that the constants $C_{\omega, m}$ of (2) are allowed to grow at a rate which is less than exponential in the distance of the $n_{\omega, m}$ 's from the origin. SULE is closely related to a dynamical condition, which we call semiuniform dynamical localization (SUDL)

$$
\sup _{t}\left|e^{-i t H_{\omega}}(n, \ell)\right| \leq \tilde{C}_{\omega, \epsilon} e^{\epsilon|\ell|} e^{-\tilde{A}|n-\ell|} .
$$

We have proven that (3) implies (4) with $A$ arbitrarily close to $\tilde{A}$, and that if $H_{\omega}$ has simple eigenvalues [6],

(C) 1995 The American Physical Society 
then (4) implies (3) with $A=\frac{1}{2} \tilde{A}$ [7]. Condition (4) is sufficient to show that $\left\langle x^{2}(t)\right\rangle$ (or any other positive moment of $x$ ) is bounded. By standard probability arguments, (4) is implied by

$$
\mathrm{E}\left(\sup _{t}\left|e^{-i t H_{\omega}}(n, \ell)\right|\right) \leq C e^{-\tilde{A}|n-\ell|},
$$

where $\mathrm{E}(\cdot)$ denotes expectation over realizations. Condition (5) has been proven by Delyon, Kunz, and Souillard [8] in the one-dimensional case and by Aizenman [9] in multidimensional cases at large coupling [10].

A priori, one may wish to consider a more restrictive condition than (3), which is to consider (2), but with $C_{\omega}$ independent of $m$ instead of $C_{\omega, m}$. We call this condition uniformly localized eigenvectors (ULE). Indeed, ULE is related to the dynamical condition [which we call uniform dynamical localization (UDL)]

$$
\sup _{t}\left|e^{-i t H_{\omega}}(n, \ell)\right| \leq \tilde{C}_{\omega} e^{-\tilde{A}|n-\ell|}
$$

in essentially the same way that SULE is related to (4). The problem is that ULE does not occur: We have shown [1,11] that ULE cannot occur for a large class of models, and, in particular, it cannot occur for the Anderson model in any dimension. It is an open question, in fact, whether there is any Schrödinger operator with ULE. The Maryland model [12], which has an unbounded quasiperiodic potential, exhibits a weak form of ULE in the sense that for any finite energy interval there exists a uniform constant for all eigenvectors with energies in this interval. The almost Mathieu operator [13], however, does not have ULE $[1,11]$. For the Anderson model, or any other random Hamiltonian obeying Eq. (5), one can actually show stronger uniformity than what is given by Eq. (3). That is, one can get an explicit (dimension dependent) polynomial bound on the growth of the $C_{\omega, m}$ 's. Our definition of SULE by (3) attempts to get, at least roughly, a minimal uniformity requirement that would still have a two-way relationship with corresponding dynamical localization.

Our second set of results concerns the following discovery of Gordon [14] and del Rio, Makarov, and Simon [15]: Let $H_{\omega}$ be an Anderson Hamiltonian in the localized regime and let [16] $H_{\omega}(\lambda)=H_{\omega}+\lambda|0\rangle\langle 0|$. Then for a set $S$ of couplings $\lambda$, which is dense and locally uncountable [17], $H_{\omega}(\lambda)$ has a purely singular continuous spectrum [18]. In particular, for $\lambda \in S,\left\langle x^{2}(t)\right\rangle$ is unbounded [19]. So, the strong dynamical localization discussed above can be destroyed by an arbitrarily small perturbation of the potential at a single point; a disturbing fact.

We have found [1] that this instability is a mild one in the following senses: (i) For all $\lambda,\left\langle x^{2}(t)\right\rangle \leq C(\ln |t|)^{2}$ for $t$ large. (ii) The spectral measures in the singular continuous case are supported on a set of zero Hausdorff dimension [20]. In fact, this follows from (i) by a result of Last [21], which is based on ideas originally due to Guarneri [22]. (iii) $S$ is contained in a set of coupling constants $\tilde{S}$ so that $\tilde{S}$ has zero Hausdorff dimension, and so that if $\lambda \notin \tilde{S}, H_{\omega}(\lambda)$ has pure point spectrum.

We would like to thank J. Avron and A. Klein for useful discussions. The work of S. J. was supported by the National Science Foundation under Grant No. DMS9208029. The work of B.S. was supported by the National Science Foundation under Grant No. DMS9401491.

[1] R. del Rio, S. Jitomirskaya, Y. Last, and B. Simon, "Operators with Singular Continuous Spectrum, IV. Hausdorff Dimensions, Rank One Perturbations, and Localization" (to be published).

[2] The results hold for much more general distributions; see Ref. [1].

[3] Or complete in some energy range.

[4] For $\delta=0$, it is known that the limit is zero in the region of pure point spectrum. See B. Simon, Commun. Math. Phys. 134, 209-212 (1990).

[5] S. Aubrey and G. Andre, Ann. Israel Phys. Soc. 3, 133164 (1980); J. Avron and B. Simon, Duke Math. J. 50, 369-391 (1983).

[6] This is known in the Anderson localization regime, see B. Simon, Rev. Math. Phys. 6, 1183-1185 (1994).

[7] The idea behind the proof that (4) implies (3) is to use simplicity of the spectrum and (4) to show that

$$
\left|\varphi_{\omega, m}(n)\right|\left|\varphi_{\omega, m}(\ell)\right| \leq \tilde{C}_{\omega, \epsilon} e^{\epsilon|\ell|} e^{-\tilde{A}|n-\ell|}
$$

Take $n_{\omega, m}$ to be a point where $\varphi_{\omega, m}(\cdot)$ takes its maximum value, then

$$
\left|\varphi_{\omega, m}(n)\right|^{2} \leq\left|\varphi_{\omega, m}(n)\right|\left|\varphi_{\omega, m}\left(n_{\omega, m}\right)\right| \leq \tilde{C}_{\omega, \epsilon} e^{\epsilon\left|n_{\omega, m}\right|} e^{-\tilde{A}\left|n-n_{\omega, m}\right|} .
$$

[8] F. Delyon, H. Kunz, and B. Souillard, J. Phys. A 16, 25 (1983).

[9] M. Aizenman, Rev. Math. Phys. 6, 1163-1182 (1994). For related results, also see F. Martinelli and E. Scoppola, Rivista Nuovo Cimento 10, (1987).

[10] If the potential is unbounded (e.g., Gaussian), there are also results at small coupling and large energy.

[11] S. Jitomirskaya, "Singular Continuous Spectrum and Uniform Localization for Ergodic Schrödinger Operators" (to be published).

[12] The Maryland model is the discrete Schrödinger operator in one dimension with potential $V(n)=\lambda \tan (2 \pi \alpha n+$ $\theta$ ). See D. R. Grempel, S. Fishman, and R. E. Prange, Phys. Rev. Lett. 49, 833 (1982); R. E. Prange, D. R. Grempel, and S. Fishman, Phys. Rev. B 29, 6500-6512 (1984); A. Figotin and L. Pastur, Commun. Math. Phys. 95, 401-425 (1984); B. Simon, Ann. Phys. 159, 157-183 (1985).

[13] The almost Mathieu operator is the discrete Schrödinger operator in one dimension with potential $V(n)=\lambda \cos (2 \pi \alpha n+\theta)$. For large $\lambda$, it is known to exhibit localization in the sense of Eq. (2); see 
Ya. Sinai, J. Phys. 46, 861-909 (1987); J. Fröhlich, T. Spencer, and P. Wittwer, Commun. Math. Phys. 132, 5-25 (1990); S. Jitomirskaya, Commun. Math. Phys. 165, 49-57 (1994).

[14] A. Gordon, Commun. Math. Phys. 164, 489-505 (1994).

[15] R. del Rio, N. Makarov, and B. Simon, Commun. Math. Phys. 165, 59-67 (1994).

[16] $H_{\omega}(\lambda)$ has the potential modified at zero by a small amount $\lambda$. For a general theory on such perturbations, see B. Simon, in Spectral Analysis of Rank One Perturbations and Applications, Lecture notes given at the Vancouver Summer School in Mathematical Physics, August 1993.

[17] Technically, $S$ is a dense $G_{\delta}$.
[18] Singular continuous spectrum is sometimes called "critical states" in physics literature.

[19] It follows from the RAGE theorem, see M. Reed and B. Simon, Methods of Modern Mathematical Physics, III. Scattering Theory (Academic Press, London, 1979), that if $\left\langle x^{2}(t)\right\rangle$ is bounded, then the spectrum is pure point. Also see Ref. [21].

[20] For a discussion of the Hausdorff dimension, see K. J. Falconer, Fractal Geometry (Wiley, Chichester, 1990).

[21] Y. Last, "Quantum Dynamics and Decompositions of Singular Continuous Spectra" (to be published).

[22] I. Guarneri, Europhys. Lett. 10, 95-100 (1989). 